\title{
Solid State Plasma in the Pulse Modulated Magnetic Field
}

\author{
Z. Jankauskas* ${ }^{*}$ S. Barauskas and V. Kvedaras \\ Department of Electrical Engineering, Vilnius Gediminas Technical University \\ Saulëtekio av. 11, LT-10223 Vilnius, Lithuania
}

\begin{abstract}
Radio frequency (RF) magnetoplasmic waves known as helicons will propagate in solid-state plasma of semiconductors when a strong magnetic field is applied. Helicons have an exact analogy with an electromagnetic whistler wave which is frequently propagated in the low density plasma of the Earth ionosphere. In our experiments the modulated magnetic field is being used for excitation of helicons. It is shown that in the case of pulse modulated field along with the RF helicon waves the transient cyclotron frequency oscillations exist in the semiconductor plasma. For observation of the cyclotron radiation frequency $\omega_{c}$ the modulation depth about one percent is sufficient. The measurement of $\omega_{c}$ provides an opportunity to determine the masses of electrons and holes in solid-state plasma of semiconductors. In already existing method the absorption of electromagnetic radiation on the cyclotron frequency is being used. For this case it is necessary to have an external infrared generator. In our method the cyclotron radiation is being generated by electrons (or holes) directly and additional external excitation is not needed.
\end{abstract}

PACS numbers: 40.41.Cr, 41.20.Jb

\section{Introduction}

The plasmas that exist in solids offer a unique opportunity to observe plasma behaviour under well-defined and accurately known conditions. In a solid, one can usually determine quite precisely the number of charge carriers, their masses, their random heat energy and the boundaries of the plasma. For example, radio frequency magnetoplasmic waves known as helicons [1] will propagate in solid-state plasma of semiconductors when a strong magnetic field is applied. Helicons have an exact analogy with an electromagnetic whistler wave which is frequently propagated in the low density plasma of the Earth ionosphere. In our experiments the modulated magnetic field is being used for excitation of helicons. It is shown that in the case of pulse modulated field along with the RF helicon waves the transient cyclotron frequency oscillations exist in the semiconductor plasma. For observation of the cyclotron radiation frequency $\omega_{c}$ the modulation depth about one percent is sufficient. The measurement of $\omega_{c}$ provides an opportunity to determine the charge carriers masses in the solid-state plasma of semiconductors.

\section{Geometry of experimental device}

The semiconductor sample $(n$-InSb) is in the form of plate [2]. The strong magnetic field $H$ is directed along the axis $z$ and is perpendicular to the sample plane $x-y$. The modulation depth of $H$ varies from 0 to 10 percent. The sample dimensions in $x-y$ directions are much larger than the sample thickness. In this case the

* corresponding author; e-mail: Zigmantas.jankauskas@el.vtu.lt electromagnetic wave (helicon) propagating in semiconductor is a circularly polarized plane wave of the form $\exp (\mathrm{i}(-k z+\omega t))$ with the components only $x$ and $y$.

\section{Equations of motion for one-component plasma}

Assuming that the conductivity of the experimental plate is provided by the electrons with an isotropic mass we have the following equations of motion for the electrical current components $j_{x}$ and $j_{y}$ (Gauss units)

$$
\begin{aligned}
& \frac{\mathrm{d}}{\mathrm{d} t} j_{x}+\gamma j_{x}+\frac{e H}{m c} j_{y}=\frac{N e^{2}}{m} E_{x}, \\
& \frac{\mathrm{d}}{\mathrm{d} t} j_{y}+\gamma j_{y}-\frac{e H}{m c} j_{x}=\frac{N e^{2}}{m} E_{y},
\end{aligned}
$$

where $N, e, m$ and $\gamma$ are the density, charge, mass and reciprocal of collision time of the electrons, respectively $E_{x}$ and $E_{y}$ are the components of varying electrical helicon field, and $H$ is the strong magnetic field along the axis $z, c$ - velocity of light.

Multiplying Eq. (2) by i and adding to Eq. (1) we obtain for the circularly polarized wave

$$
\begin{aligned}
& j_{x}+\mathrm{i} j_{y}=I \mathrm{e}^{\mathrm{i} \omega t}, \quad E_{x}+\mathrm{i} E_{y}=E \mathrm{e}^{\mathrm{i} \omega t}, \\
& \frac{\mathrm{d}}{\mathrm{d} t}\left(I \mathrm{e}^{\mathrm{i} \omega t}\right)+\left(\gamma-\mathrm{i} \omega_{c}\right) I \mathrm{e}^{\mathrm{i} \omega t}=\frac{N e^{2}}{m} E \mathrm{e}^{\mathrm{i} \omega t},
\end{aligned}
$$

$$
\begin{aligned}
& \frac{\mathrm{d}}{\mathrm{d} t} I+\left[\gamma+\mathrm{i}\left(\omega-\omega_{c}\right)\right] I=\frac{N e^{2}}{m} E, \\
& \frac{\mathrm{d}}{\mathrm{d} t} I+[\gamma+\mathrm{i} \Delta \omega] I=\frac{N e^{2}}{m} E, \quad \Delta \omega=\omega-\omega_{c}, \\
& \omega_{c}=\frac{e H}{m c}
\end{aligned}
$$$$
\text { where }
$$

is the cyclotron frequency of the electron, and $I$ and $E$ 
are the amplitudes of circularly polarized wave, $\omega-\mathrm{RF}$ frequency electrical field.

\section{The influence of pulse modulated magnetic field}

Pulse modulated strong magnetic field $H$ may be written in the form

$$
H=H_{0}+h u(t),
$$

where time function $u(t)$ represents a square wave of rectangular pulses

$$
\begin{aligned}
& u(t)=1 \text { for } \quad 2 n T<t<(2 n+1) T, \\
& u(t)=0 \text { for } \quad(2 n+1) T<t<2(n+1) T, \\
& n=0,1,2,3, \ldots
\end{aligned}
$$

For simplicity the pulse duration and the interval between pulses are assumed equal to $T$.

Waveforms like (8) and (9) are very common in digital circuits [3].

In accordance with Eqs. (7)-(9) and (6)

$$
\begin{aligned}
& H=\left(H_{0}+h\right)=H_{1} \\
& \text { for } \quad 2 n T<t<(2 n+1) T, \\
& H=H_{0}=H_{2} \\
& \text { for } \quad(2 n+1) T<t<2(n+1) T, \\
& \omega_{c}=\omega_{c 1}=\frac{e H_{1}}{m c} \text { for } 2 n T<t<(2 n+1) T, \\
& \omega_{c}=\omega_{c 2}=\frac{e H_{2}}{m c}
\end{aligned}
$$$$
\text { for } \quad(2 n+1) T<t<2(n+1) T \text {. }
$$

The solution of Eq. (5b) for current amplitude $I$ is correspondingly

$$
\begin{aligned}
& I=\frac{N e^{2}}{m} E \frac{1}{\gamma+\mathrm{i} \Delta \omega_{1}}\left[1-A_{1} \exp \left(-\left(\gamma+\mathrm{i} \Delta \omega_{1}\right) t\right)\right] \\
& \quad \text { for } \quad 2 n T<t<(2 n+1) T \\
& I=\frac{N e^{2}}{m} E \frac{1}{\gamma+\mathrm{i} \Delta \omega_{2}}\left[1-A_{2} \exp \left(-\left(\gamma+\mathrm{i} \Delta \omega_{2}\right) t\right)\right] \\
& \quad \text { for } \quad(2 n+1) T<t<2(n+1) T \\
& \Delta \omega_{1}=\omega-\omega_{c 1}, \quad \Delta \omega_{2}=\omega-\omega_{c 2},
\end{aligned}
$$

where $A_{1}$ and $A_{2}$ are functions of $\Delta \omega_{1}, \Delta \omega_{2}, \gamma$ and $T$. In the case when

$$
T \geq 3 \gamma^{-1}
$$

we have

$$
A_{1}=\frac{\mathrm{i}\left(\Delta \omega_{2}-\Delta \omega_{1}\right)}{\gamma+\mathrm{i} \Delta \omega_{2}}, \quad A_{2}=\frac{\mathrm{i}\left(\Delta \omega_{1}-\Delta \omega_{2}\right)}{\gamma+\mathrm{i} \Delta \omega_{1}} .
$$

Thus, the current amplitude $I$ alongside with the constant part contains the oscillatory transients of the frequencies $\Delta \omega_{1}=\omega-\Delta \omega_{c 1}$ and $\Delta \omega_{2}=\omega-\Delta \omega_{c 2}$. The helicon frequency $\omega$ is of RF range and $\omega_{c 1}$ and $\omega_{c 2}$ are of infrared range. Therefore, practically $\Delta \omega_{1}$ and $\Delta \omega_{2}$ coincides with the corresponding cyclotron frequencies, Eq. (16). If $\Delta \omega_{2}=0$ and $\Delta \omega_{1} \gg \gamma$, then for the time $t=\pi / \Delta \omega_{c 1}$ the current $I$ has the value of opposite sign in comparison to its initial value for $t=0$. Consequently, Eq. (5b) and its solutions (14), (15) describe also the well-known effect of density inversion when conductivity becomes negative.

It should be noted that Eqs. (5a, b) contain only the difference of the frequencies $\omega$ and $\omega_{c}$, e.g. the frequency modulation of helicon wave and modulation of magnetic field are equivalent.

The transient oscillations of the semiconductor plasma's response with the cyclotron frequency were observed experimentally [4] for the modulation depths of the magnetic field about one percent, e.g. for $h / H_{0}=0.01$, see Eq. (7).

The observation of cyclotron radiation is effective especially if the detected response signal is proportional to the time derivative of $I$ when the signal becomes much stronger (proportional to $\omega_{c}$ ).

The real part of the ratio $I / E$ is equal to the conductivity of semiconductor plasma and the imaginary part is responsible for helicon wave propagation (effective dielectrical constant). We have from Eqs. (14), (15)

$$
\begin{aligned}
& \operatorname{Re}\left(\frac{I}{E}\right)=\frac{N e^{2}}{m} \frac{1}{\gamma^{2}+\Delta \omega_{1}^{2}} \\
& \quad \times\left\{\gamma+\frac{\Delta \omega_{2}-\Delta \omega_{1}}{\gamma+\Delta \omega_{2}^{2}}\left[\left(\gamma^{2}-\Delta \omega_{1} \Delta \omega_{2}\right) \sin \Delta \omega_{1} t\right.\right. \\
& \left.\left.\quad-\gamma\left(\Delta \omega_{1}+\Delta \omega_{2}\right) \cos \Delta \omega_{1} t\right] \exp (-\gamma t)\right\}, \\
& \operatorname{Im}\left(\frac{I}{E}\right)=-\frac{N e^{2}}{m} \frac{1}{\gamma^{2}+\Delta \omega_{1}^{2}} \\
& \quad \times\left\{\Delta \omega_{1}+\frac{\Delta \omega_{1}-\Delta \omega_{2}}{\gamma+\Delta \omega_{2}^{2}}\left[-\gamma\left(\Delta \omega_{1}+\Delta \omega_{2}\right) \sin \Delta \omega_{1} t\right.\right. \\
& \left.\left.\quad+\left(\Delta \omega_{1} \Delta \omega_{2}-\gamma^{2}\right) \cos \Delta \omega_{1} t\right] \exp (-\gamma t)\right\}, \\
& \quad \text { for } \quad 2 n T<t<(2 n+1) T,
\end{aligned}
$$

and

$$
\begin{aligned}
& \operatorname{Re}\left(\frac{I}{E}\right)=\frac{N e^{2}}{m} \frac{1}{\gamma^{2}+\Delta \omega_{2}^{2}} \\
& \quad \times\left\{\gamma+\frac{\Delta \omega_{1}-\Delta \omega_{2}}{\gamma^{2}+\Delta \omega_{1}^{2}}\left[\left(\gamma-\Delta \omega_{1} \Delta \omega_{2}\right) \sin \Delta \omega_{2} t\right.\right. \\
& \left.\left.\quad-\gamma\left(\Delta \omega_{1}+\Delta \omega_{2}\right) \cos \Delta \omega_{2} t\right] \exp (-\gamma t)\right\}, \\
& \operatorname{Im}\left(\frac{I}{E}\right)=-\frac{N e^{2}}{m} \frac{1}{\gamma^{2}+\Delta \omega_{2}^{2}} \\
& \quad \times\left\{\Delta \omega_{2}+\frac{\Delta \omega_{2}-\Delta \omega_{1}}{\gamma^{2}+\Delta \omega_{1}^{2}}\left[-\gamma\left(\Delta \omega_{1}+\Delta \omega_{2}\right) \sin \Delta \omega_{2} t\right.\right. \\
& \left.\left.\quad+\left(\Delta \omega_{1} \Delta \omega_{2}-\gamma^{2}\right) \cos \Delta \omega_{2} t\right] \exp (-\gamma t)\right\}, \\
& \quad \text { for } \quad(2 n+1) T<t<2(n+1) T .
\end{aligned}
$$

Equtions (19) $-(22)$ show that semiconductor plasma's conductivity and effective dielectrical constant are modulated by the cyclotron frequency. 


\section{Conclusions}

In the pulse modulated magnetic field the semiconductor plasma response alongside with the traditional RF helicon wave contains the transients oscillating with cyclotron frequency, which can be observed experimentally.

Physically it means that the semiconductor conductivity and effective dielectrical constant are modulated with the same cyclotron frequency of infrared range.

By the measurement of cyclotron frequency the masses of electrons and holes can be determined on the basis of formula (6).

In already existing methods the absorption of electromagnetic radiation on the cyclotron frequency is being used. For this case it is necessary to have an external infrared generator. In our method the cyclotron radiation is being generated by electrons (or holes) directly and additional external excitation is not needed.

\section{References}

[1] See, for example, A. Libchaber, R. Veilex, Phys. Rev. 127, 774 (1962).

[2] Z. Jankauskas, L. Laurinavičius, Electronics and Electrical Engineering 37, 32 (2002).

[3] C.R. Paul, S.A. Nasar, L.E. Unnewehr, Introduction to Electrical Engineering, McGraw-Hill, New York 1992 , p. 771.

[4] Z. Jankauskas, V. Kvedaras, L. Laurinavičius, Physica B 346-347, 539 (2004). 